\title{
MULTIFOCAL EPITHELIAL HYPERPLASIA: REPORT OF TWO CASES
}

\author{
Małgorzata Radwan-0czko', Aleksandra Sender-Janeczek², Maciej Kaczorowski ${ }^{3}$, Marta Szczepaniak', Agnieszka Hałoń ${ }^{3}$ \\ 'Department of Oral Pathology, Wroclaw Medical University, Wroclaw, Poland \\ ${ }^{2}$ Department of Periodontology, Wroclaw Medical University, Wroclaw, Poland \\ ${ }^{3}$ Department of Pathomorphology and Oncological Cytology, Wroclaw Medical University, Wroclaw, Poland
}

\begin{abstract}
Multifocal epithelial hyperplasia, also known as Heck's disease, is not a common pathology, which is well-known to most physicians. Therefore, asymptomatic proliferation of the oral mucosa has been reported in the literature with differences in various geographic regions. Here, we report unique cases of multifocal epithelial hyperplasia involving multiple sites of oral cavity in a 59-year-old male and a 9-year-old boy.
\end{abstract}

KEY wORDs: multifocal epithelial hyperplasia, human papillomavirus, Heck's disease.

J Stoma 2019; 72, 6: 278-281

DOI: https://doi.org/10.5114/jos.2019.93797

\section{INTRODUCTION}

Multifocal epithelial hyperplasia (MEH), also known as Heck's disease, was firstly described in Indian children in 1965 by Archard et al. Although this asymptomatic proliferation of the oral mucosa has been reported in the whole world, it is still not well-known to most physicians. It is characterized by the presence of multiple slowgrowing, papulo-nodular, elevated, and smooth-surface lesions. Usually, they are round or oval, well-defined nodules with color similar to normal mucosa, and measuring from 0.1 to $0.5 \mathrm{~cm}$ in diameter. $\mathrm{MEH}$ is more common in females, and the most affected locations are labial, lingual, and buccal mucosas. The lesions are painless, not ulcerated, or inflamed. They do not transfer into carcinoma and no case of malignance has been reported in the literature. Focal epithelial hyperplasia (FEH) is associated with human papillomaviruses (HPV) subtypes 13 and 32 infection, however, other HPV genotypes have been also detected in MEH lesions. Poverty, malnutrition, close living conditions, immunodeficiency, and human immunodeficiency virus (HIV) infection are listed among the essential epidemiological factors. In addition, genetic susceptibility to HPV infection associated with HLA types has recently been reported. It can be present both in young and old patients, with higher incidence in females [1-7].

\section{CASE PRESENTATION}

\section{CASE 1}

A 59-year-old male patient reported to the Department of Periodontology and Oral Pathology of Wroclaw Medical University for a check-up. He had no history of previous systemic disease and was not under any medications. The patient did not complain of any symptoms. He was a smoker, and extra-oral examination demonstrated normal, healthy, and dark complexion skin. Intra-oral examination revealed multiple papillary lesions involving buccal

\section{JOURNAL OF} STOMATOLOGY 

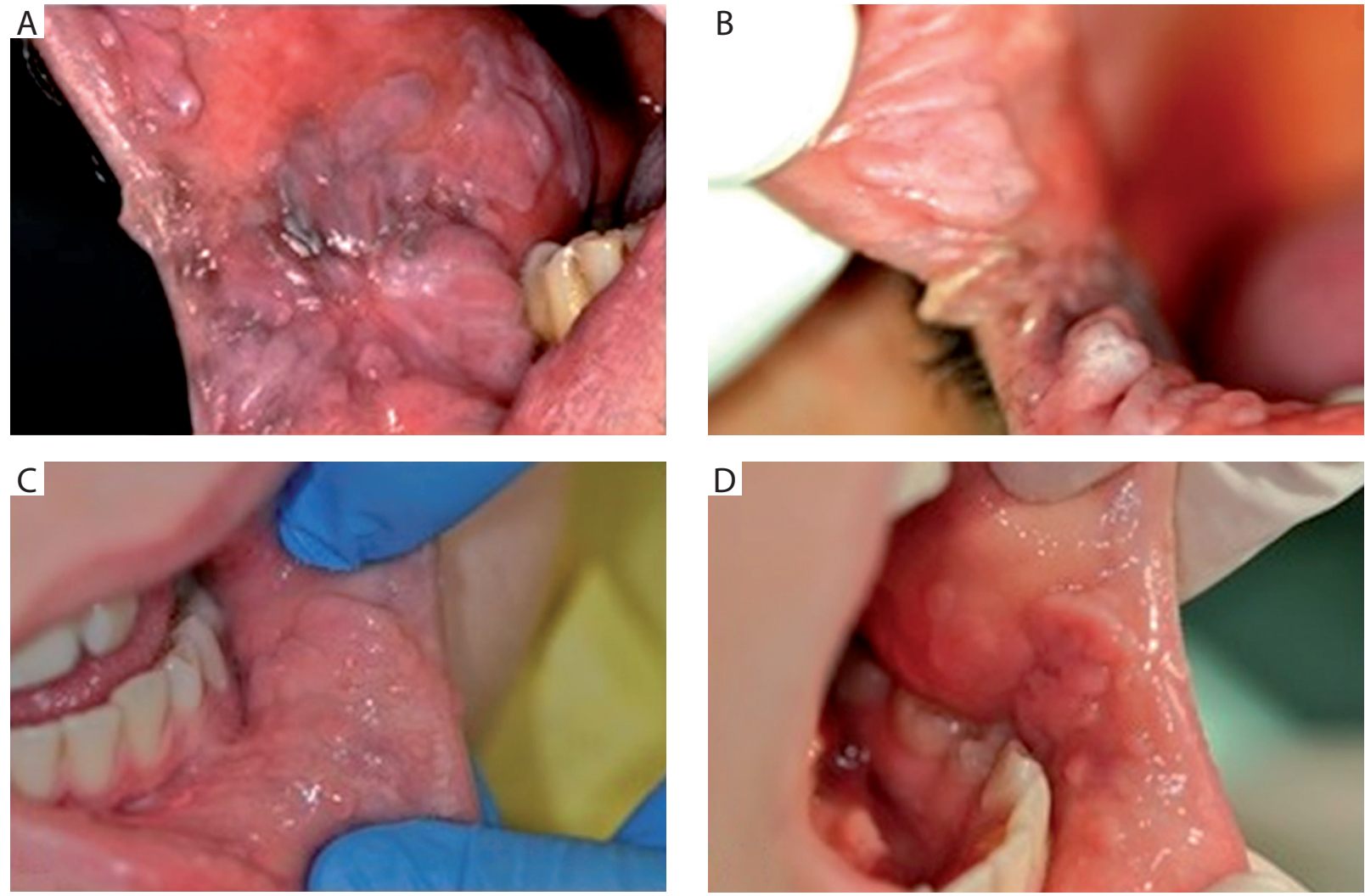

FIGURE 1. Clinical presentation of multifocal epithelial hyperplasia (MEH) of a 59-year-old patient. A) Clinical presentation at first visit. B) Clinical presentation 6 months after an improvement of oral condition. C, D) Clinical presentation of MEH of a 9-year-old patient

mucosa on both sides and intra-oral side of the lips (Figure $1 \mathrm{~A}, \mathrm{~B})$. The buccal mucosa seemed to be swollen and was light-brown pigmented. The examination using chemiluminescent light and 1\% tolonium chloride (Visilite) revealed no abnormalities of mucosal hyperplastic lesions.

The patient was asked to quit smoking and to remove some traces of dental roots. Due to the presence of dental calculus, scaling and polishing were performed. Based on the clinical features, a provisional diagnosis of HPVassociated oral mucosa disease was established.

On the next visit, after local anesthesia, a biopsy was performed, which included partial excision of buccal mucosa.

Microscopic evaluation showed thickened, acanthotic epithelium with prominent parakeratosis. Rete ridges were elongated, clubbed, and formed horizontal anastomoses. Numerous koilocytes and some mitosoid bodies were found. Ki67 staining revealed mildly elevated proliferative activity of the epithelium (Figure 2).

The patient was also asked to visit his GP doctor and conduct a general health check-up, blood tests, and lungs X-ray examination. All investigations revealed no abnormalities. We advised the patient to have better oral hygiene and to use vitamin A topically, 3 times a day. On the next controlled visits, the local status was unchanged and the patient did not complain of any problems.

\section{CASE 2}

A 9-year-old boy was referred to the Department of Periodontology and Oral Pathology of Wroclaw Medical University (Figure 1 C, D). Patient's mother did not report of any systemic diseases regarding her son, and physical examination showed no significant abnormality. Intra-oral examination revealed multiple elevated papules on the left buccal and lower lip mucosa. All lesions were asymptomatic and not inflamed. Moreover, the patient associated appearance of the lesions included occlusal trauma of the labial and lips mucosa. Routine blood investigations revealed no abnormality. Using local anesthesia, biopsy of the lesions on the left buccal mucosa was performed.

Histopathological examination showed considerable thickening of squamous epithelium with parakeratosis and acanthosis as well as marked elongation and bridging of rete pegs. Characteristic mitosoid bodies and focal koilocytosis were present.

Immunohistochemical staining for Ki67 demonstrated increased basal and suprabasal reactivity, therefore confirming the hyperplastic nature of lesions. Labelling for p53, a nuclear protein frequently overexpressed in neoplastic conditions, resulted as low (Figure 3). 

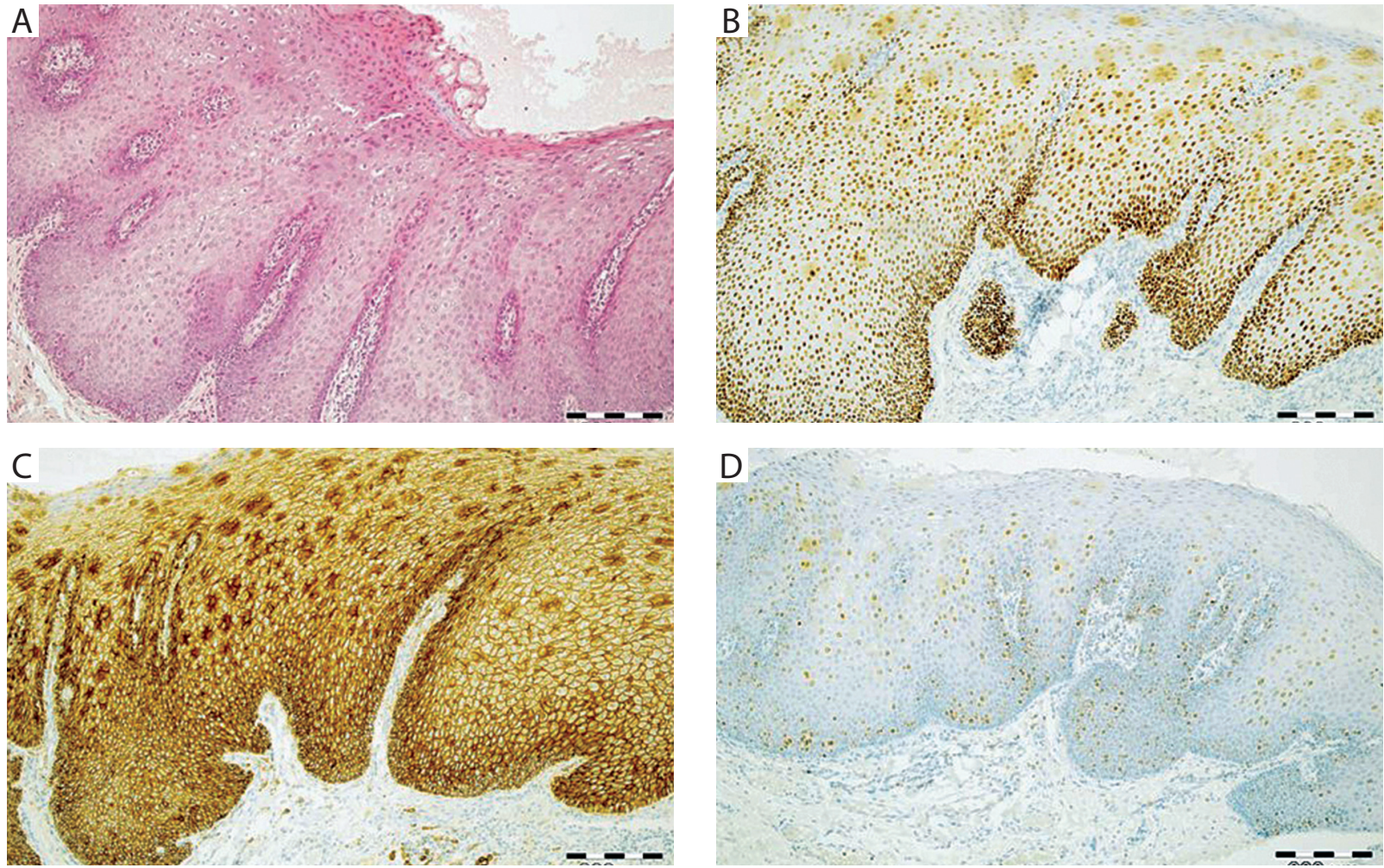

FIGURE 2. Thickened buccal epithelium with striking parakeratosis and numerous koilocytes in the upper layers, H\&E stain. A) Strong staining for squamous epithelial markers: nuclear p63 (B) and membranous CD138, which also highlights a few subepithelial plasma cells (C). D) Reaction with anti-Ki67-positive cells scattered throughout the basal and spinous layers
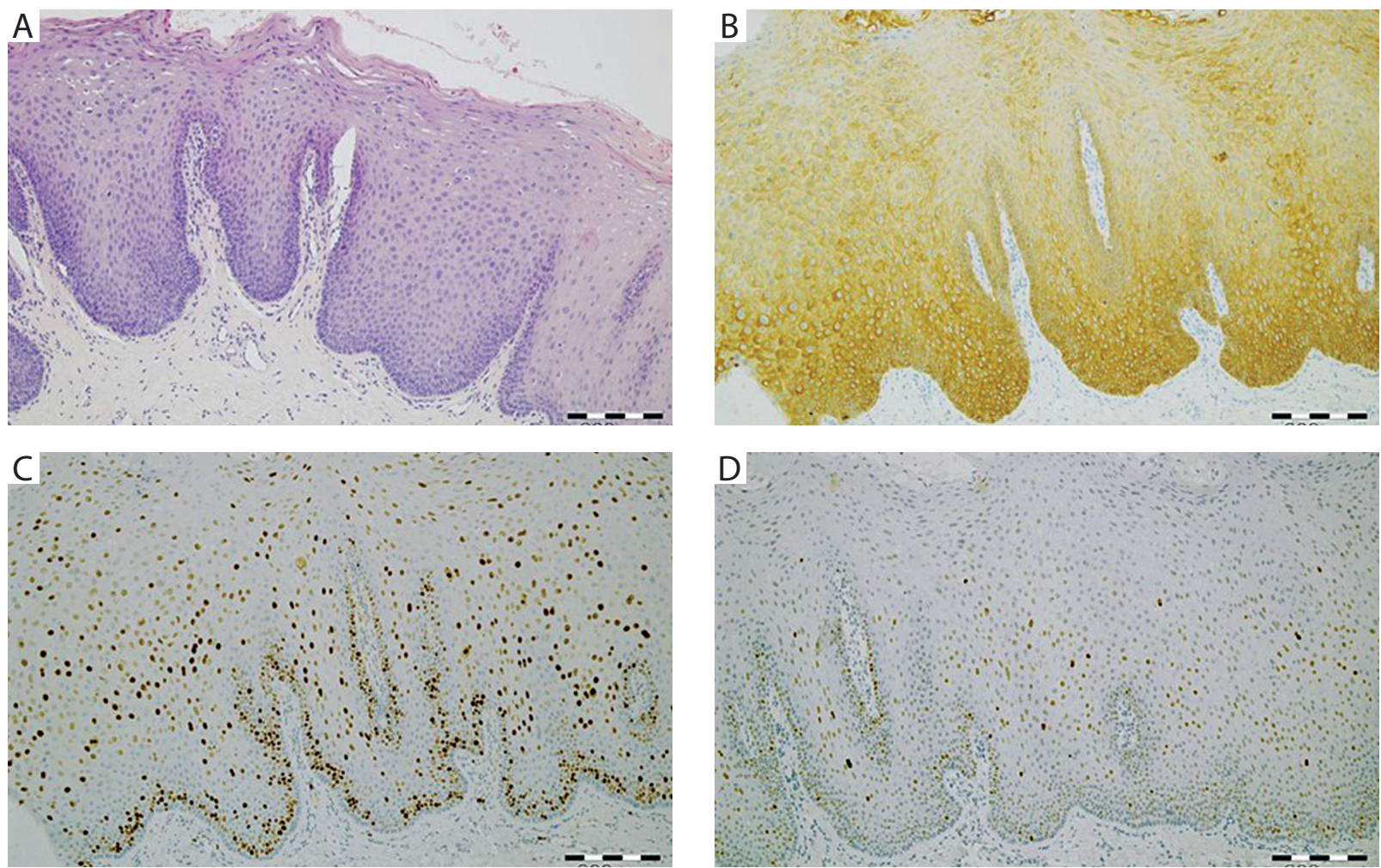

FIGURE 3. Hyperplastic squamous epithelium with mitosoid bodies and groups of koilocytes, H\&E stain. A) Epithelial thickening is highlighted by cytoplasmic reaction with anti-CK5/6 antibody. B) Nuclear positivity for Ki67 in basal and suprabasal layers of the epithelium confirms the hyperplastic character of the lesion. C) Low p53 expression in scattered keratinocytes (D) 


\section{DISCUSSION}

FEH predominantly affects females who are between the $1^{\text {st }}$ and $2^{\text {nd }}$ decades of life. In the literature, cases of young people such as 17-year-old girl from Ecuador [8], 21-year-old American Indian women [9], and 15-yearold boy from Lithuania [10] have been described. There were also cases of $\mathrm{MEH}$ reported in children. Agnew et al. described a case of a five-year-old girl who presented with multiple intra-oral lesions on the buccal mucosa and tongue, which regressed spontaneously in 15 months [11]. Gökahmetoğlu et al. described FEH in Turkish family, with 13-year-old daughter, 35-year-old mother, and 40-year-old father showing the same histological and virological features in examination, probably because of HPV transmission [12].

However, Park et al. reported a case of FEH located on one side of the attached gingiva from the canine to the second upper molar and mandible first molar of a 53-year-old Korean man associated with a metal-ceramic bridge. The authors did not detect any HPV subtype in histological examination [13].

Although MEH tends to occur in the first two decades of life, it can be encountered in elderly patients as well. Shamloo et al. described a case of a 92-year-old man who presented with multiple, asymptomatic, circumscribed, soft, flattened papules in different sizes on the retrocommissure of lower lip [14].

Biopsy is the gold standard for the final diagnosis of FEH. Histological examination reveals thickened squamous epithelium with marked acanthosis and parakeratosis, but no cytologic atypia. Rete ridges are elongated and frequently club-shaped [15]. Mitosoid bodies, HPV-altered keratinocytes with hyperchromatic and clumped nuclei resembling mitotic figures are considered the most specific microscopic features of FEH. Typical koilocytes with vacuolated cytoplasm and pyknotic nuclei are also present [16].

Treatment is generally not necessary because it is a self-limiting disease and lesions usually disappear with age. Hence, we advised periodic long-term follow-ups to monitor the status of lesion. Nonetheless, different therapeutic procedures have been reported such as surgical excision, laser ablation, cryotherapy, electrocauterization, interferon, retinoic acid, and $\mathrm{CO}_{2}$ laser surgery $[17,18]$.

\section{CONFLICT OF INTEREST}

The authors declare no potential conflicts of interest with respect to the research, authorship, and/or publication of this article.

\section{References}

1. Ozden B, Gunduz K, Gunhan O, Ozden FO. A case report of focal epithelial hyperplasia (Heck's disease) with PCR detection of human papillomavirus. J Maxillofac Oral Surg 2011; 10: 357-360.
2. Said AK, Leao JC, Fedele S, Stephen R, Porter SR. Focal epithelial hyperplasia - an update. J Oral Pathol Med 2013; 42: 435-442.

3. Lopez-Jornet P, Camacho-Alfonso F, Berdugo L. Oral focal epithelial hyperplasia. NY State Dent J 2010; 76: 54-55.

4. Gültekin SE, Tokman Yildirim B, Sarisoy S. Oral focal epithelial hyperplasia: report of 3 cases with human papillomavirus DNA sequencing analysis. Pediatr Dent 2011; 33: 522-544.

5. Patterson AT, Andritsos L, Allen CM, Gru A, Kaffenberger BH. Multifocal epithelial hyperplasia (Heck disease) in a patient with chronic lymphocytic leukemia. J Cutan Pathol 2014; 41: 694-696.

6. Probhat MPV, Lakshmi CR, Sai Madhavi N, Bhavana AM, Sarat G, Ramamohan K. Multifocal epithelial hyperplasia of oral cavity expressing HPV 16 gene: a rare entity. Case Rep Dent 2013; 2013: 871306.

7. Akoğlu G, Metin A, Ceylan GG, Emre S, Akpolat D, Süngü N. Focal epithelial hyperplasia associated with human papillomavirus 13 and common human leukocyte antigen alleles in a Turkish family. Int J Dermatol 2015; 54: 174-178.

8. Vera-Iglesias E, García-Arpa M, Sánchez-Caminero P, RomeroAguilera G, Cortina de la Calle P. Focal epithelial hyperplasia. Actas Dermosifiliog 2007; 98: 621-623.

9. Durso BC, Pinto JMV, Jorge JJr, Paes de Almeida O. Extensive focal epithelial hyperplasia: case report. J Can Dent Assoc 2005; 71: 769-771.

10. Puriene A, Rimkevicius A, Gaigalas M. Focal epithelial hyperplasia: case report. Stomatologija 2011; 13: 102-104.

11. Agnew C, Alexander S, Prabhu N. Multifocal epithelial hyperplasia. J Dent Child (Chic) 2017; 84: 47-49.

12. Gökahmetoğlu S, Ferahbaș A, Canöz Ö. Focal epithelial hyperplasia in a Turkish family. Infez Med 2014; 4: 322-325.

13. Park MW, Cho YA, Kim SM, Myoung H, Lee JH, Lee SK. Focal epithelial hyperplasia arising after delivery of metal-ceramic fixed dental prosthesis. J Adv Prosthodont 2014; 6: 555-558.

14. Shamloo N, Mortazavi H, Taghavi N, Baharvand M. Multifocal epithelial hyperplasia: a forgotten condition in the elderly. Gen Dent 2016; 64: 72-74.

15. Archard HO, Heck JW, Stanley HR. Focal epithelial hyperplasia: an unusual oral mucosal lesion found in Indian children. Oral Surg Oral Med Oral Pathol 1965; 20: 201-212.

16. Carlos R, Sedano HO. Multifocal papilloma virus epithelial hyperplasia. Oral Surg Oral Med Oral Pathol 1994; 77: 631-635.

17. Ruiz R, Silva GR, Menchaca HR. Focal epithelial hyperplasia. Lancet 2014; 384: 173.

18. van der Voort EA, Arani SF, Hegt VN, van Praag MC. Focal epithelial hyperplasia of the oral mucosa. A unique manifestation of human papillomavirus. Ned Tijdschr Tandheelkd 2009; 116: 149-151. 